Int. J. Dev. Biol. 55: 583-590

doi: $10.1387 /$ ijdb.103275ct

\title{
Regulation and activity of JNK signaling in the wing disc peripodial membrane during adult morphogenesis in Drosophila
}

\author{
CHATURVEDULATRIPURA ${ }^{*}, 1$, NULU-PRAFULLA CHANDRIKA ${ }^{1}$, \\ VUTUKURU-NAGALAKSHMI SUSMITHA ${ }^{1}$, STÉPHANE NOSELLI ${ }^{2}$ and LS SHASHIDHARA ${ }^{1,3}$ \\ ${ }^{1}$ Centre for Cellular and Molecular Biology, Hyderabad, India, '2Institut Biologie du Développement et Cancer', Univ. \\ Nice Sophia-Antipolis, CNRS, France and ${ }^{3}$ Indian Institute of Science Education and Research, Pune, India
}

\begin{abstract}
Thorax closure in Drosophila is a process during adult morphogenesis in which the anterior ends of the presumptive notum of the two wing imaginal discs fuse to make a seamless thorax. Similar to dorsal closure during embryogenesis, this process is regulated by Decapentaplegic and JNK signaling pathways. Despite the fact that Peripodial Membrane (PM) cells do not contribute to the formation of any adult structure, they are known to facilitate the process of thorax closure. Here we show that JNK signaling is activated only in a subset of PM cells, known as medial edge cells. While the mechanism that activates JNK signaling specifically in the medial edge cells of the PM is still not understood, the results presented here show that the pair rule gene odd skipped is required to ensure that JNK signaling is not activated anywhere else in the wing disc. Medial edge cells of the PM are elongated in shape, while the remaining PM cells are hexagonal. Down regulation of JNK signaling in the medial edge cells results in defective thorax closure in adult flies. It also causes the transformation of the morphology of medial edge cells into hexagonal shape. Conversely, activation of JNK signaling in hexagonal cells of the PM causes transformation of their morphology to elongated shape.Thus, similar to dorsal closure during embryogenesis, JNKmediated elongation of medial edge cells is functionally correlated to the process of thorax closure.
\end{abstract}

KEY WORDS: odd-skipped, puckered, thorax closure, epithelium, medial edge cells

\section{Introduction}

Epithelia are integral components of tissues and organs in all metazoa. During development, epithelial sheets undergo various types of morphogenesis in which, cells, either individually or in groups, follow a coordinated pattern of division, constriction, elongation and/or migration. These distinct, but, general cell behaviors underlie the assembly of majority of organs and tissues in multicellular organisms. In some normal and pathological circumstances (metastasis), epithelial cells can undergo a transition towards a mesenchymal invasive state.

Thorax closure is an important event during adult morphogenesis in Drosophila and is analogous to embryonic dorsal closure (Fristrom and Fristrom, 1993). While the two wing imaginal discs (which give rise to both the wing proper and the notum) develop and differentiate independently, during metamorphosis, the thoracic tissues fuse to make one seamless T2 thorax. Each wing imaginal disc is made up of a columnar epithelium or disc proper (DP) that will give rise to most of the adult structures, and a squamous peripodial epithelium or peripodial membrane (PM), which contributes very little to adult tissues. During metamorphosis, cells of PM may play an active role through the dramatic change of their shape, either by intensive stretching or contraction (Fristrom and Fristrom, 1993, Milner et al., 1984). Earlier reports have established a signaling role for PM in patterning DP during wing morphogenesis (Cho et al., 2000; Gibson and Schubiger, 2000; Gibson et al., 2002; Pallavi and Shashidhara, 2003, 2005; Pastor-pareja et al., 2004).

During thorax closure, the leading edge cells of the two imaginal discs are specified and they execute coordinated forward movements by dramatically changing their shape (either contraction or stretching) and move over the substratum till the opposing sides of the two discs meet (Martin-Blanco et al., 2000). They then fuse in the antero-dorsal midline forming a seamless joint to form

Abbreviations used in this paper: DP, disc proper; PM, peripodial membrane.

\footnotetext{
*Address correspondence to: ChaturvedulaTripura. Centre for Cellular and Molecular Biology, Hyderabad 500007, India. e-mail: tripura@ccmb.res.in
} 

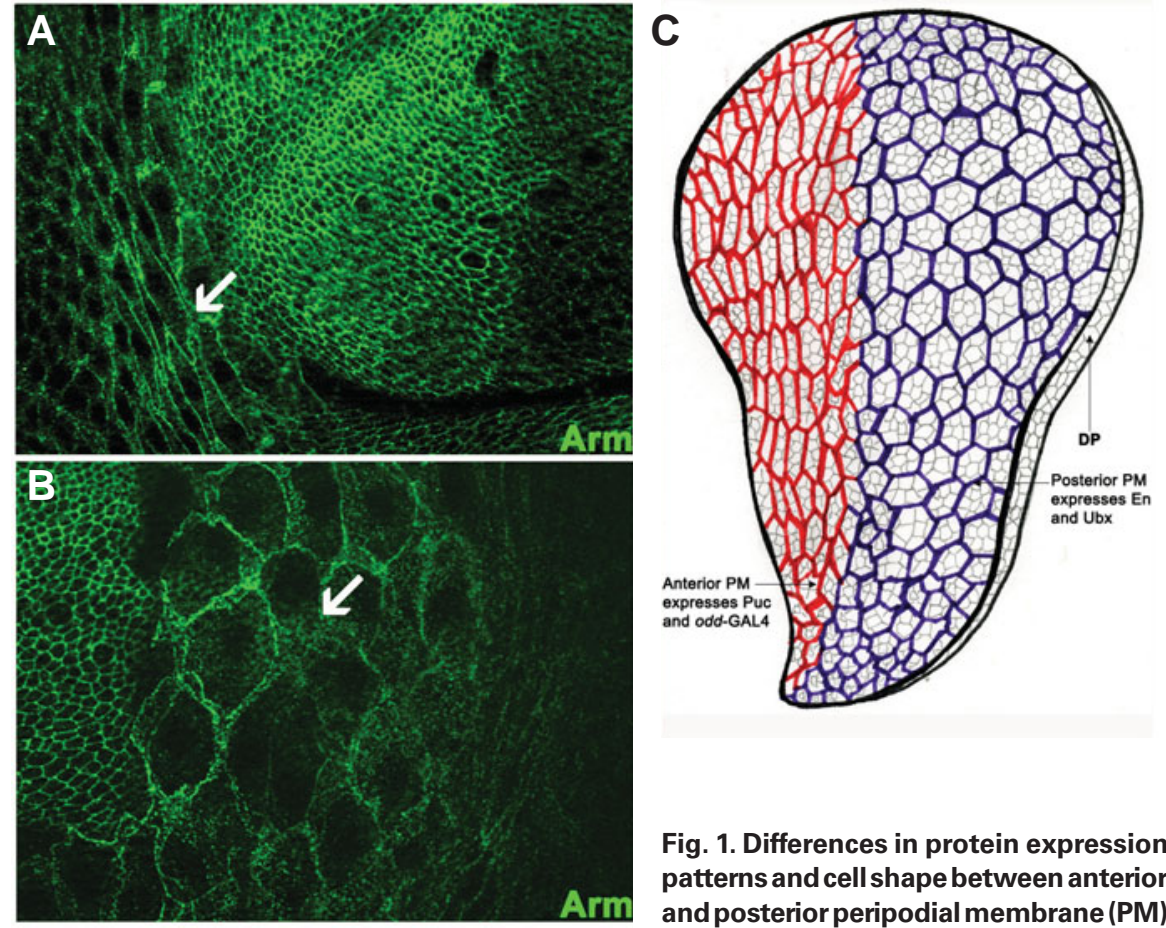

Fig. 1. Differences in protein expression patterns and cell shape between anterior and posterior peripodial membrane (PM) cells. (A,B) Wildtype wing imaginal disc stained for Armadillo (Arm). Arm is localized to the intracellular surface of adherens junctions and thus, marks the outline of epithelial cells. Note long elongated cells in (A) and large hexagonal cells in (B) (arrows), which represent anterior and posterior compartments of PM, respectively. Small hexagonal cells in both $(A, B)$ are disc proper cells. (C) Schematic figure to show the organization of disc proper (DP) and PM cells. DP cells are shown in grey as small hexagonal cells. Overlaying these cells are large PM cells. The anterior cells in the PM are elongated (red) and the posterior ones are hexagonal (b/ue). In this figure and in all subsequent figures wing imaginal discs are shown with anterior to the left and the posterior to the right. one contiguous T2 thorax. Similar to dorsal closure, thorax closure is also characterized by prominent actin organization at the midline of fusion (Zeitlinger and Bohmann, 1999). The entire process is mediated by changes in cell shape and there is no cell proliferation or cell recruitment. With a network of several molecules involved, the process of thorax closure in Drosophila provides a good genetic model system for the analysis of the mechanisms involved in coordinating epithelial sheet spreading and cell recognition during wound healing and development (Martin-Blanco et al., 2000; Agnes et al., 1999).

Earlier reports from one of our labs (SN) have shown that JNK pathway is involved in thorax closure (Agnes et al., 1999; Noselli and Agnes, 1999). JNK signaling plays an essential role in the process of thorax closure by mediating disc morphogenesis, spreading, and cell fate determination (Agnes et al., 1999). Flies mutant in JNK signaling fail in the process of thorax closure giving rise to adults with thorax cleft phenotypes (Agnes et al., 1999; Noselli and Agnes, 1999; Zeitlinger and Bohmann, 1999; Martin-Blanco et al., 2000; Suzanne et al., 2001; Tateno et al., 2000; Ishimaru et al., 2004). JNK signaling is an intracellular relay pathway regulating fundamental biological events such as proliferation, differentiation, morphogenesis and apoptosis in response to numerous inputs (Davis, 2000; Chang and Karin, 2001; Weston and Davis, 2002). JNK
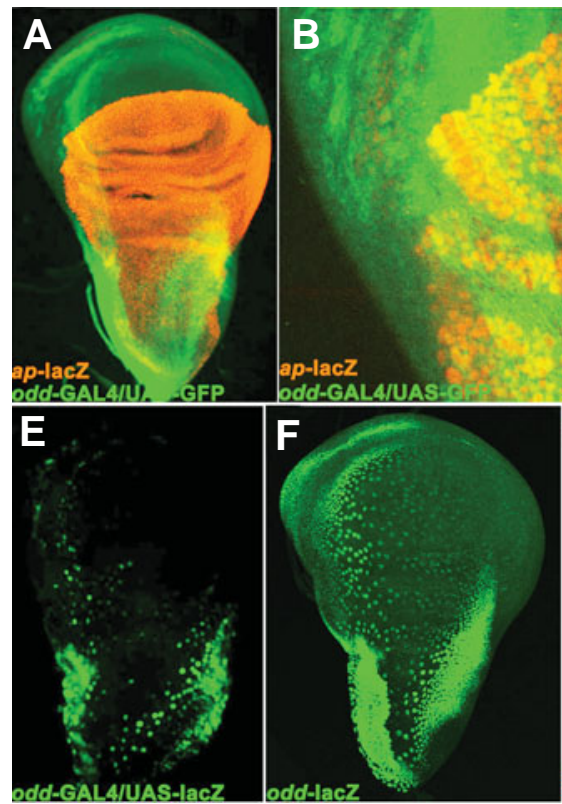
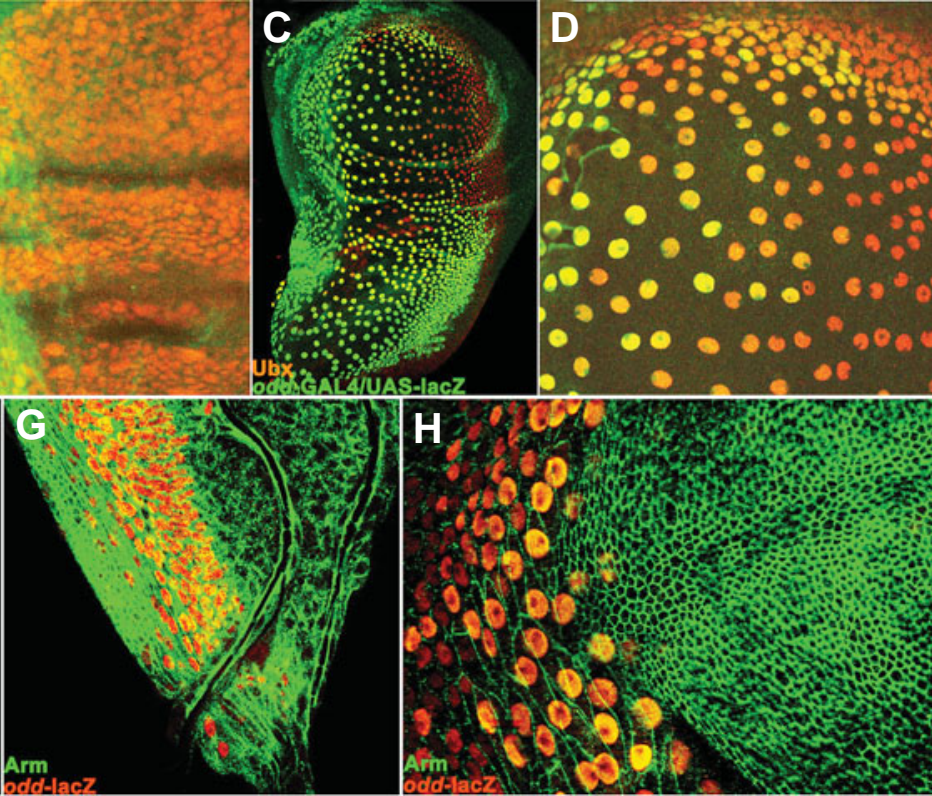

Fig. 2. odd-GAL4 and odd-lacZ are specific to elongated medial edge cells of the peripodial membrane of the wing disc. (A) odd-GAL4/UASGFP; ap-lacZ wing discs stained for $\beta$-galactosidase (red) and GFP (green). (B) A part of the disc in (A) at higher magnification. Note that odd-GAL4 and ap-lacZ expressions do not overlap in any part of the wing disc, an indication that odd-GAL4 is PM-specific. (C) Odd-GAL4/UAS-nuclear lacZ wing disc stained for $\beta$-galactosidase (green) and Ubx (red). Note, while

both expressed in PM, the two proteins are expressed in two different spatial domains. odd-GAL4 is expressed in the anterior compartment and Ubx in the posterior compartment. (D) A part of the disc in (C) at higher magnification. (E) Odd-GAL4/UAS-FLP; Act5C>stop>lacZ wing disCS showing a representative clone generated as part of lineage analysis of odd-GAL4. This clone and all other clones ( $n=10)$ were PM-specific. (F) Odd-lacZ wing imaginal disc stained for $\beta$-galactosidase. (G,H) Higher magnification images of odd-lacZ wing discs stained for $\beta$-galactosidase (red) and Arm (green). (G) Region near the notum and $\mathbf{( H )}$ near the pouch. All three images show that odd-lacZ expression, similar to odd-GAL4, is PM-specific. 
is necessary to maintain the adhesion of the imaginal leading edge cells to their larval substrate and to promote actin dynamics (Martin-Blanco et al., 2000). JNK signaling in conjunction with the AP-1 activity is not only involved in thorax closure, but more generally in the assembly and morphogenesis of imaginal discs (Zeitlinger and Bohmann, 1999).

At the core of the JNK cascade are the stress-activated kinases JNKK and JNK (Ip and Davis, 1998), the Drosophila homologues of which are encoded by hemipterous (hep) and basket (bsk), respectively. Hep phosphorylates and thereby activates Bsk, which in turn activates DJun/Fos by phosphorylation (Glise et al., 1995; Riesgo-Escovar et al., 1996; Sluss et al., 1996). Finetuning of the JNK signaling is maintained by puckered (puc), a dual specificity phosphatase, at the level of JNK/bsk by a feed back mechanism (Martin-Blanco et al., 1998). Puc not only acts as a buffer by stabilizing JNK activity at a medium level but also helps in repressing JNK activity when it is no longer required (Zeitlinger and Bohmann, 1999). Thus, Puc is a reliable marker to trace spatio-temporal regulation of JNK activity.

Here we have explored the link between JNK signaling and $\mathrm{PM}$ in the process of thorax closure. JNK signaling is active only in a subset of PM cells, known as medial edge cells, located in its anterior compartment. We show that this spatially restricted activity of JNK signaling plays an important role in determining elongated shape of medial edge cells and in regulating thorax closure. Our data support a role for the pair rule gene odd-skipped (odd) in keeping JNK signaling off from all cells of the wing disc including the medial edge cells of the PM. A hitherto unknown mechanism overrides this negative regulation of Odd in the medial edge cells, thus restricting JNK activity to only those cells.

\section{odd-GAL4 expression in the peripodial membrane}

We have previously reported that odd-GAL4 expression in the wing disc is PM-specific (Pallavi and Shashidhara, 2003). oddGAL4 expression does not overlap with ap-lacZ (DP specific), providing evidence that odd-Gal4 is specific to the PM (Fig. 2A,B). Detailed analysis of its expression suggested that odd-GAL4 is expressed strongly in the anterior medial edge cells and in few cells in the posterior notum. Its expression does not overlap with Ubx expression, which is expressed in the posterior cells of the PM (Fig. 2 C,D). Extensive lineage analysis experiments also suggested that odd-GAL4 is expressed strongly in the anterior PM cells (Fig. 2E). Another independent enhancer-trap line odd-lacZ was also found to be expressing in the medial edge cells and in few cells in the posterior notum (Fig. 2F), but not expressed in hexagonal posterior cells or the pouch cells (Fig. $2 \mathrm{G}, \mathrm{H}$ ).

We examined the expression pattern of Odd using antibodies against the protein, which faithfully reproduces pair rule expression pattern in the embryos (Kosman et al., 1998; Fig. 3A). Though odd-Gal4 expression was restricted to the PM, interestingly, endogenous Odd is expressed in all cells of the wing disc (Fig. 3B). This suggests that odd-GAL4 is a mere enhancer-trap that captures a subset of Odd expression pattern.

\section{Loss of odd leads to thoracic defects}

Above observations suggested that although Odd is expressed in all cells of the wing discs, it has an enhancer, which is PMspecific. We, therefore, investigated if loss of function of odd has any effect on thorax closure. We generated transgenic flies that enable tissue-specific knock-down of Odd using the UAS-GAL4 system to study the role of Odd in wing disc morphogenesis.

\section{Results}

We had earlier reported that the PM of the wing disc is divided into three spatial domains based on the expression patterns of various genes. A posterior domain that expresses the Hox gene Ultrabithorax (Ubx) and the segment polarity gene engrailed (en), an A/P boundary which is a narrow stripe of decapentaplegic $(d p p)$-expressing cells and an anterior domain that does not express any of these genes (Pallavi and Shashidhara, 2005). Anterior cells of the PM are more elongated (Fig. 1A). As these cells make first contact with the opposite wing disc, they are generally referred to as medial edge cells. The majority of Ubx-expressing posterior cells in the centre of the wing pouch are hexagonal (Fig. 1B). A schematic figure on the organization of PM and DP and the two major types of cells are shown in Fig. $1 \mathrm{C}$.

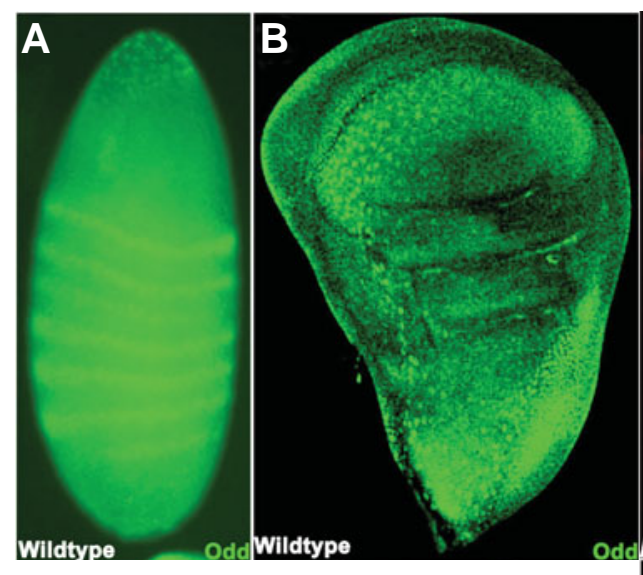

Fig. 3. RNAi-mediated knock-down of Odd. $(A, B)$ Wildtype embryo (A) and wingdisc (B) stained for Odd using anti-Odd antibodies. While Odd is expressed in odd-numbered parasegments in the embryo, it is expressed in almost all cells of both $P M$ and DP in wing discs. (C) Pnr-GAL4/UAS-lacZ wing disc stained for $\beta$-galactosidase showing the expression pattern of the GAL4 driver. (D) Pnr-GAL4/ UAS-odd ${ }^{\text {RAi }}$ wing disc stained for Odd using antiOdd antibodies. (E) Da-GAL4/UAS-lacZ wing disc stained for $\beta$-galactosidase showing the expression pattern of the GAL4 driver. (F) Da-GAL4/UAS-odd RNA
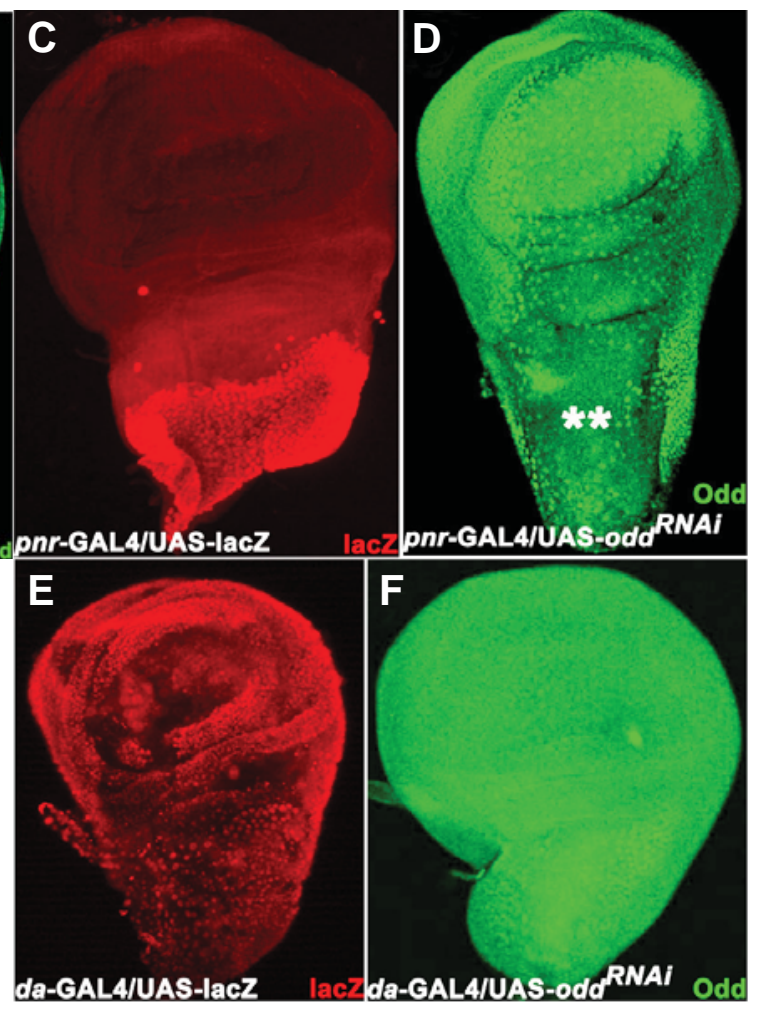

wing disc stained for Odd using anti-Odd antibodies. Note, RNAi-mediated knock-down of Odd expression in D (asterisks) and $F$ (the entire disc) in the regions where RNAi expression is driven. 
Fig. 4. Down regulation of odd expression causes defective thorax closure. (A-D) Wildtype (A), da-GAL4/UAS-odd ${ }^{\text {RNAi }}$ (B), en-GAL4/ $U A S-$ odd $^{\text {RNAi }}$ (C) andpnr-GAL4/UAS-odd ${ }^{\text {RNAi }}$ (D) adult flies. Note severe (with da and en-GAL4 drivers) to mild (with pnr-GAL4 driver) phenotypes associated with UAS-odd ${ }^{\mathrm{RNAi}}$-mediated knockdown of odd expression ( $>>25)$. Images in $\left(A^{\prime}-D^{\prime}\right)$ show scanning electronic microscope images of the phenotypes corresponding to the genotype $(A-D)$, respectively.

We observed down regulation of Odd levels in the regions where UAS-odd ${ }^{\text {RNA }}$ expression was induced (Fig. 3 D,F).

First we examined what happens if PM-specific expression of Odd is knocked-down. RNAi-mediated knock down of Odd in PM using odd-GAL4 did not cause any cellular or adult thoracic phenotype. We then examined the effect of down regulation of Odd using a variety of GAL4 drivers. Amongst the several GAL4 drivers tried, thorax
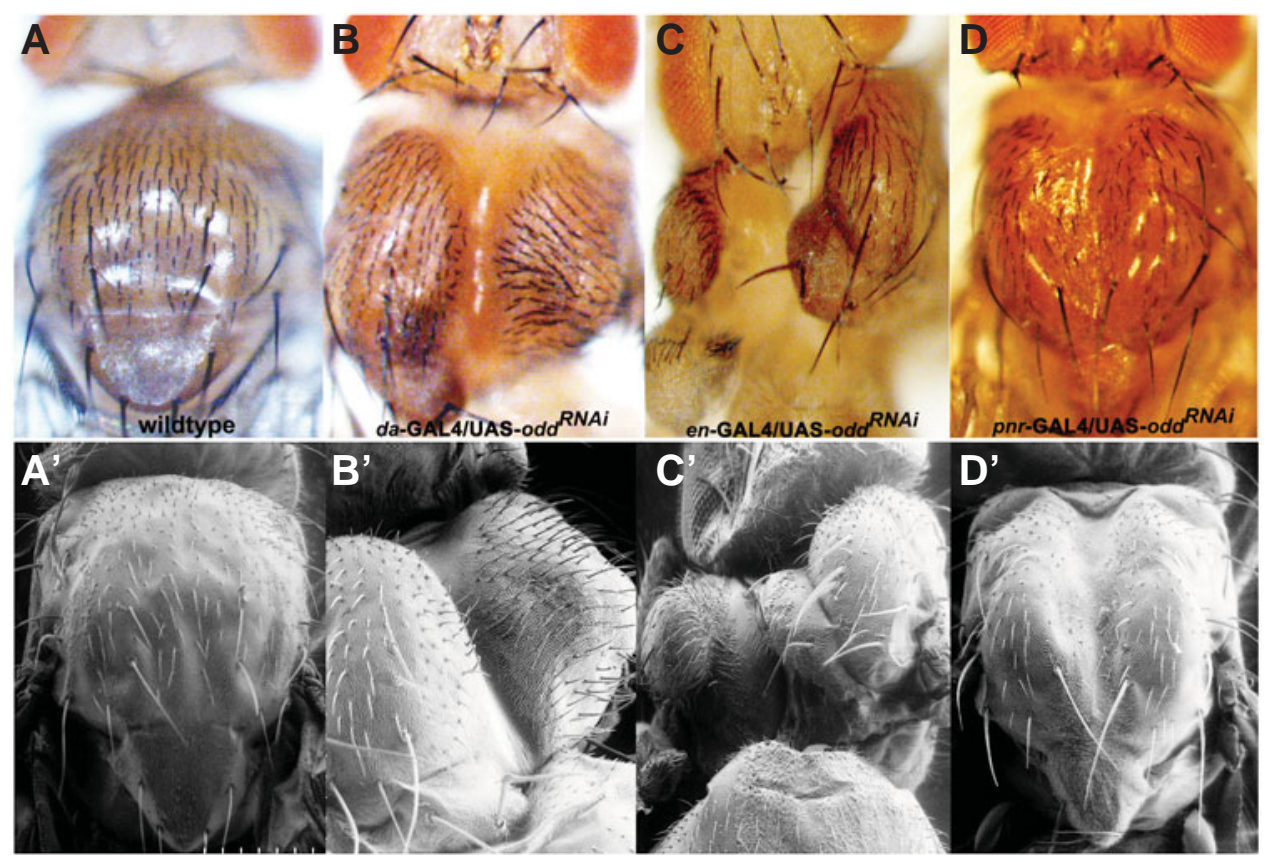

closure defects were observed when odd is knocked down by driving the expression of UAS-odd ${ }^{R N A i}$ with da-GAL4, en-GAL4 and pnr-Gal4 drivers (Fig. 4 B-D). da-GAL4 is expressed in all the cells of PM and DP (Fig. 3C), while pnr-GAL4 is expressed in the presumptive notum and in a small number of PM cells overlaying notum (Fig. 3E). en-GAL4 is also expressed in both PM and DP, although only in the posterior cells (Pallavi and Shashidhara, 2003, 2005). Knock down of Odd with da-GAL4 resulted in more than $70 \%$ pupal lethality. Both surviving adults and lethal pupae showed strong thorax closure defects (Fig. 4B). Loss of Odd driven with en-Gal4 resulted in only $10 \%$ pupal lethality, but with strong thorax defects. Majority of the flies that

emerged showed thorax defects with one side of the thorax either completely deformed or absent (Fig. 4C). Although no lethality was associated with pnr-GAL4 driven down regulation of odd, mild thoracic closure defects were observed (Fig. 4D). Down regulation of Odd only in the PM or DP using GAL4 drivers that are either PM specific (such as Ubx-GAL4, odd-GAL4, pucGal4) or DP specific (such as MS1096-GAL4) did not cause any thorax phenotype (data not shown). It is, therefore, likely that down regulation of Odd in both the medial edge cells and the presumptive notum of PM and DP appears to be essential to induce defective thorax phenotypes since it is these cells that play a role in the thorax formation.

Fig. 5. Effect on Puc expression and adult phenotype by down regulation or ectopic activation of JNK signaling in the medial edge cells and posterior domain of wing disc, respectively. $(A, B)$ puc-lacZ (A) and odd-GAL4/UASGFP; puc-lacZ (B) wing imaginal discs stained for $\beta$-galactosidase ( $B$ is also stained for GFP). Similar to odd-GAL4 driver, puc-lacZ is mainly expressed in the medial edge cells. (C,D) Ubx-GAL4/puc-lacZ; UAS$B^{k^{w t}}$ (C) and odd-GAL4/puc-lacZ; UAS-bsk ${ }^{\mathrm{DN}}$ (D) wing imaginal discs stained for $\beta$-galactosidase. Note up-regulation of puc-lacZ levels when JNK activity is ectopically activated in posterior cells of PM and its down regulation when JNK activity is down regulated in medial edge cells (n>30). (E-G) Wildtype (E) odd-GAL4/puc-lacZ; UAS-bsk ${ }^{D N}$ (F) and odd-GAL4/puc-lacZ; UASPuc (G) adult flies showing defects in thorax closure $(n>30)$.
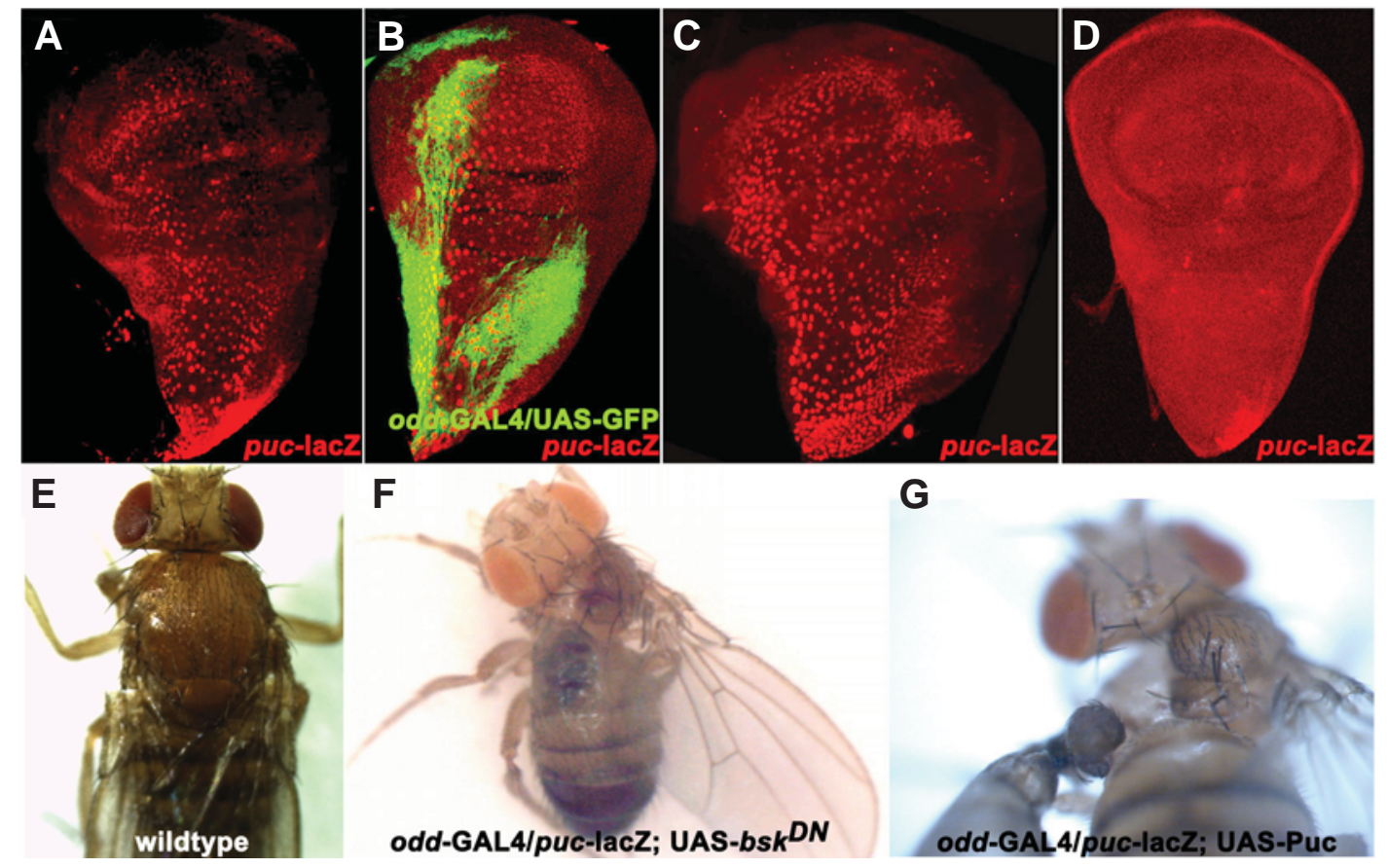

$\mathbf{F}$

G
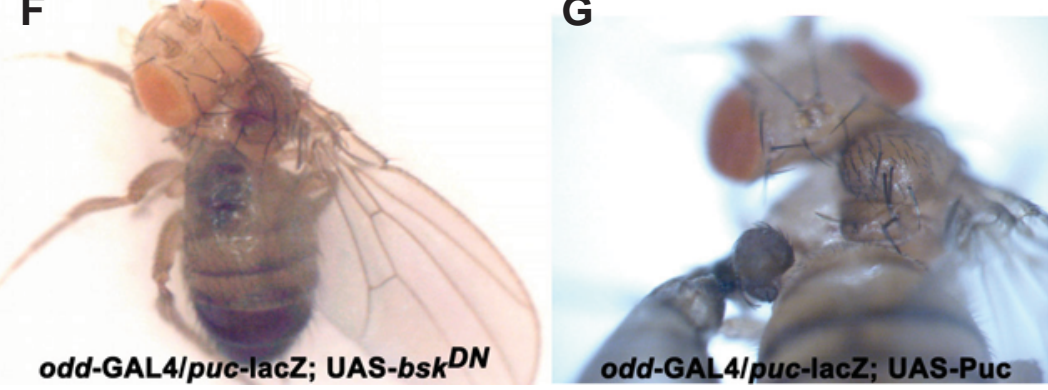


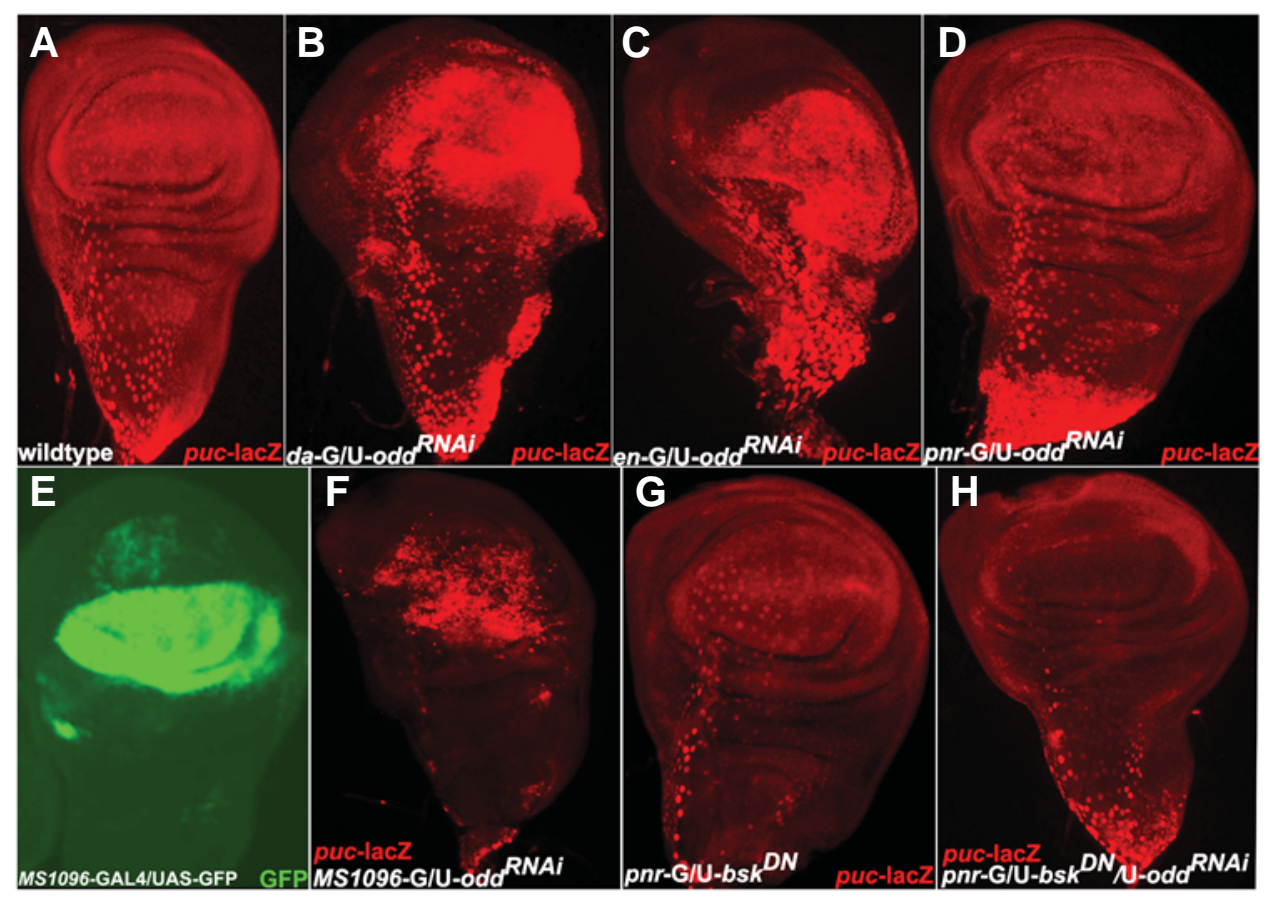

Fig. 6. Down regulation of odd causes ectopic activation of puc expression. All the discs in this figure are stained for $\beta$-galactosidase. (A) puc-lacZ wing imaginal disc. (B-D) da-GAL4/ puc-lacZ; UAS-oddRNAi (B), en-GAL4/puc-lacZ; UAS-odd ${ }^{\mathrm{RNAi}}$ (C) and pnr-GAL4/puc-lacZ; UAS odd $^{\mathrm{RNAi}}$ (D) wing imaginal discs. Note up regulation and ectopic activation of puc-lacZ levels in all imaginal discs ( $n>20)$. Adult flies of these genotypes show thorax closure defects (see Fig. 6). (E) MS1096-GAL4/UAS-GFP wingdisc. MS1096-GAL4 driver is expressed only in the dorsal pouch of the DP. (F) MS1096-GAL4/puclacZ; UAS-odd ${ }^{\mathrm{RNA}}$ wingdisc. Note up regulation of puc expression levels in the dorsal pouch. $(\mathbf{G}, \mathbf{H})$ pnr-GAL4/puc-lacZ; UAS-bsk ${ }^{\mathrm{DN}}(\mathbf{G})$ and pnr-GAL4/puc-lacZ; UAS-bsk ${ }^{\mathrm{DN}} /$ UAS-odd $^{\mathrm{RNAi}}$ (H) wing imaginal discs. Note complete down regulation of puc-lacZ levels in (G) and partial down regulation in $H$ (compare $G$ with $A$ and $D ; n>20)$.

\section{Down regulation of JNK signaling in medial edge cells is} sufficient to disrupt thorax closure

JNK activity is necessary to maintain the adhesion of medial edge cells of the wing imaginal disc to their larval substrate and to promote actin dynamics (Martin-Blanco et al., 2000). puc, a readout of JNK signaling, is expressed exclusively in the medial edge cells of the PM (Agnes et al., 1999; Martin-Blanco et al., 2000). In mature third instar larvae, the expression of puc-lacZ is very prominent in the stalk region (where the imaginal discs connect to the larval epidermis) and in the anterior cells of the PM (Fig. $5 \mathrm{~A}$, also see below). Thus, puc-lacZ expression pattern correlates to the activity of JNK pathway in PM cells, and thus serves as a marker for JNK activity.

As one would observe, puc-lacZ and odd-GAL4 expression patterns overlap (Fig. 5B), while Ubx-GAL4 driver does not overlap with either of the two. The expression patterns of odd-GAL4 (specific to the anterior medial edge cells) and Ubx-GAL4 (specific to the posterior hexagonal cells) are, therefore, ideal to carry out certain experiments related to understanding the role of JNK pathway in thorax closure.

Ectopic expression of the positive regulators of JNK pathway such as Bsk in the posterior compartment of PM using Ubx-GAL4 driver leads to enhanced activation of puc in the posterior domain (Fig. 5C). This suggests that the absence of expression of puc in posterior PM cells is not due to its direct repression, rather it is due to the absence of any JNK activity. Conversely over expression of the negative components of JNK such as UAS-Puc or UAS-Bsk ${ }^{\mathrm{DN}}$ driven by odd-GAL4 in the JNK active anterior cells down regulated puc expression (Fig. 5D) and the adult flies were defective in thorax closure (Fig. 5 F-G), suggesting that JNK signaling indeed has a role to play in the medial edge cells to regulate the process of thorax closure.

Interestingly, while downregulation of JNK activity in medial edge cells affected both puc expression and thorax closure, over-expression of Bsk in the posterior PM cells using Ubx-GAL4 driver was only associated with increased Puc activity and no adult phenotype was observed. This suggests that the involvement of PM in adult morphogenesis is restricted to elongated medial edge cells of the anterior compartment.

\section{Spatial regulation of JNK activity}

As the function of JNK signaling in the medial edge cells is important for thorax closure, we investigated what restricts JNK activity to medial edge cells. First, we examined if Ubx negatively regulates JNK activity in non-medial edge cells of the PM. We generated mitotic clones of Ubx in the posterior cells of PM and over-expressed Ubx in medial edge cells. In both the experiments, we examined the effect of manipulating Ubx expression on Puc. However, no change in puclevels were observed (data not shown), suggesting that Ubx does not have any role in this phenomenon. This was expected as loss of Ubx is not known to have any phenotype in adult T2.

We further examined the effect of down regulation of Odd on puc

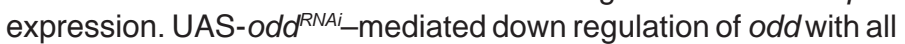
the GAL4 drivers tested showed up-regulation of puc-lacZ in both PM and DP cells, suggesting that Odd may function to down-regulate JNK pathway. Activation of puc-lacZ in the entire wing disc was seen with da-GAL4 (Fig. 6B), while it was ectopically activated in the posterior domain and in the notum when odd was down regulated with the help of en-GAL4 (Fig. 6C) and pnr-GAL4 (Fig. 6D), respectively. We also tested the effect of down-regulation of Odd using a DP-specific GAL4 driver MS1096-GAL4 (Fig. 6E). Strong activation of puc was seen in the dorsal pouch when expression of UAS-odd ${ }^{\text {PNAi }}$ was driven using MS1096-GAL4 (Fig. 6F).

Activation of puc-lac $Z$ caused by the down regulation of odd provides evidence that Odd negatively regulates JNK pathway in all regions of the wing disc, be in PM or DP. However, it was not clear if Odd regulates puc directly or by interaction with the JNK components upstream of puc. We investigated this by employing an epistasis genetic experiment, wherein both odd and bsk activi- 
ties were down regulated simultaneously. As mentioned earlier, UAS-bsk ${ }^{D N}$ mediated down regulation of Bsk activity causes reduction in puc-lacZ levels (Fig. 5D, 6G). When the expression of both UAS-odd ${ }^{R N A i}$ and UAS-bsk ${ }^{D N}$ was driven by pnr-GAL4, puc-lacZexpression was below the wildtype levels (Fig. 6H). This was significant considering the fact that UAS-odd ${ }^{\text {RNAi-mediated }}$ down regulation of Odd causes notable upregulation of puc-lac $Z$ levels. This suggests that loss of odd leads to activation of JNK pathway upstream of Bsk.

However, this poses a paradox as to why down regulation of Odd (Fig. 4 B-D) causes the same phenotype as down regulation of JNK pathway (Fig. $5 \mathrm{~F}, \mathrm{G}$ ). It is likely that both down regulation and ectopic activation of JNK pathway leads to uncoordinated movement of fusing anterior edge cells of the wing discs causing thoracic closure, which is further discussed below. Nevertheless, the results presented here suggest a negative role for Odd in restricting JNK pathway activity to a specific spatial domain. While mechanism by which JNK signaling is specifically activated in the medial edge cells of the PM is not yet understood, the results presented here show that Odd is required to ensure that JNK signaling is not activated anywhere else in the wing disc.

\section{Morphology of medial edge cells is correlated with their function}

Finally, we examined if the elongated shape of medial edge cells is correlated to the process of thorax closure. If so, this would be comparable with the observation that the leading edge cells involved in dorsal closure during embryogenesis are more elongated in shape.

Over expression of the negative components of JNK such as dominant negative Bsk in the JNK active anterior cells transformed the elongated anterior cells (Fig. 7A) into more broad hexagonal cells (Fig. 7B). Not only cells were hexagonal in shape, they appeared to be wider. Conversely, over-expression of wildtype Bsk in the posterior compartment of the wing disc driven by Ubx-GAL4 transformed the otherwise hexagonal cells in the pouch (Fig. 7C) into elongated cells (Fig. 7D). The phenotype caused by the over-expression of Bsk was not as dramatic as its loss of function in medial edge cells. The cells were mostly hexagonal, although little stretched giving the impression of elongated shape. However, loss of Ubx (in mitotic clones) from hexagonal posterior cells did not affect their shape (Fig. 7E), nor over-expression of Ubx in medial edge cells had any effect on their elongated shape (Fig. 7G). These observations again suggest requirement of JNK activity per se to determine elongated shape of PM cells. It is probable that the actin cytoskeletal network that generates necessary force for the cells to stretch and fuse with opposing cells is already (in the third instar larval stage) organized in a specific way to facilitate the fusion and this organization is dependent on JNK activity.

\section{Discussion}

Results reported here suggest that JNK pathway may have a cell autonomous activity in the medial edge cells of the PM. It is likely that the morphology and functions of medial edge cells are regulated by the JNK pathway, which is similar to the role of JNK signaling in leading edge cells of the embryo during dorsal closure. However, there is a major difference in the two. The role of medial
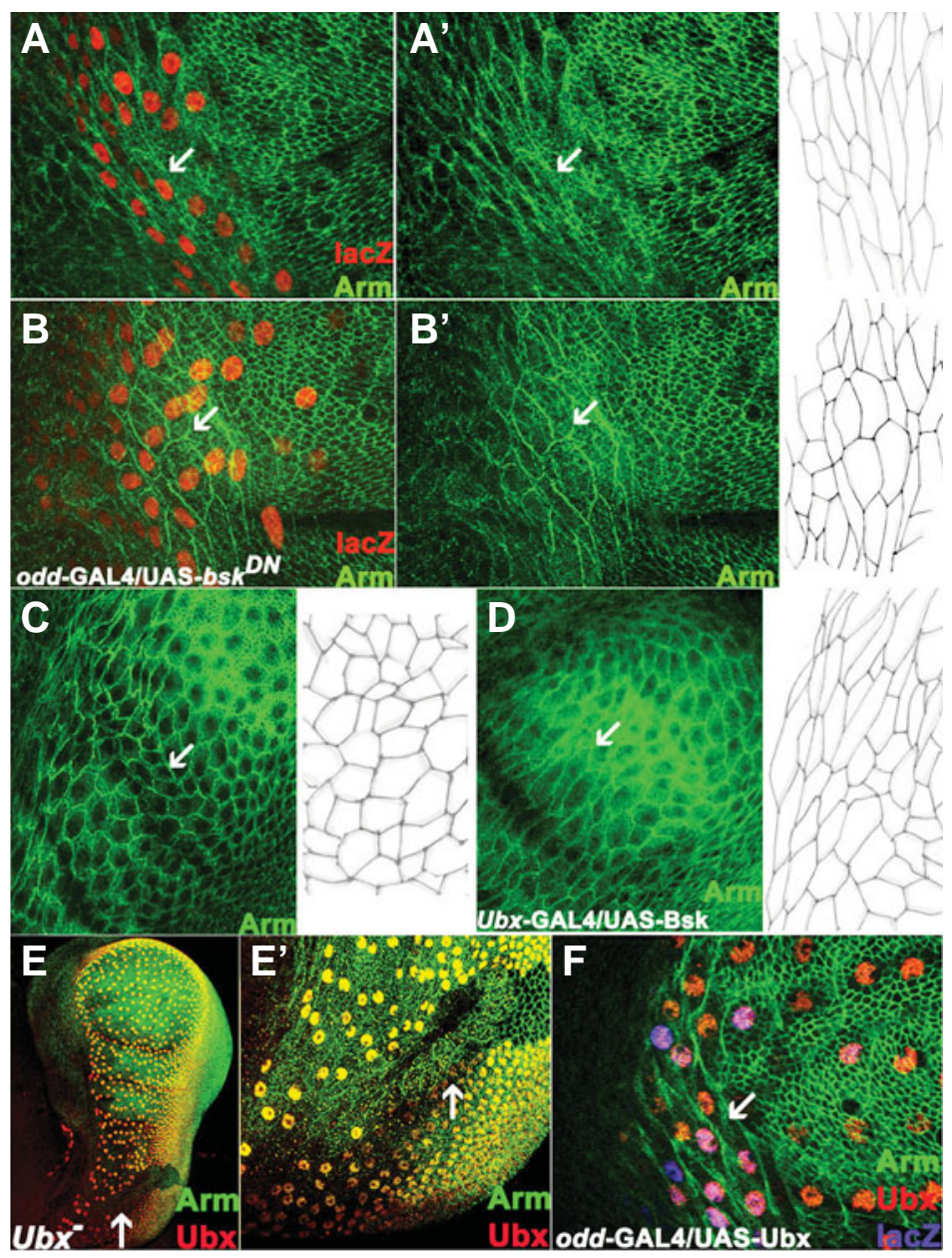

Fig. 7. Morphology of medial edge cells is dependent on JNK activity. $(\mathbf{A}, \mathbf{B})$ Wildtype (A) and odd-GAL4/UAS-nuclear lacZ; UAS-bsk ${ }^{\mathrm{DN}}$ (B) wing discs stained for $\beta$-galactosidase (red) and Arm (green). (A' ', $\mathbf{B}^{\prime}$ ) Images in $(A, B)$, respectively in single channel to highlight the cell morphology. Note transformation of elongated medial edge cells (arrow in $A, A^{\prime}$ ) to hexagonal shape (arrow in B, $\left.B^{\prime} ; n>20\right)$. (C,D) Wildtype (C) and Ubx-GAL4/UAS-Bsk ${ }^{\text {wt }}$ (D) wing discs stained for Arm. Note transformation of hexagonal posterior cells (arrow in $C$ ) to elongated shape (arrow in $D ; n>20)$. Sketches next to $\left(A^{\prime}, B^{\prime} C\right.$ and $D)$ show artist's impression of the phenotypes. These sketches, however, are not true representations. (E) hsFLP; PIFRTJUbx1 wing discs stained for Ubx (green) and Arm (red) showing a representative clone of Ubx-cells in $P M$ (arrows). (E') A part of the disc in (E) at higher magnification. Note that the morphology of Ubx-cells is unaltered ( $n=5)$. (F) Tubulin-GAL80 ts odd-GAL4/ UAS-nuclear lacZ; UAS-Ubx wing disc (from larvae grown at $18^{\circ} \mathrm{C}$ for 3 days and then shifted to $27^{\circ} \mathrm{C}$ until the wandering stage) stained for $\beta$-galactosidase (blue), Ubx (red) and Arm (green). Note that over-expression of Ubx has no effect on the morphology of medial edge cells $(n=10)$.

edge cells of the PM is restricted to bring opposing wing discs along the anterior-dorsal edges and thereby bringing underlying DP cells for fusion. By functioning from medial edge cells of the PM, JNK signaling may provide the guidance cues for proper fusion rather than directly involving in the fusion of DP cells.

The present work also suggests a role of odd in regulating JNK signaling for the formation of intact thorax during morphogenesis. Under normal conditions in the wing disc, odd probably down regulates JNK signaling in all cells and a specific activation mecha- 
nism overrides this effect such that the pathway is activated only in the medial edge cells and thus ensures normal thorax closure. Loss of odd function results in hyper activation of JNK, which is indicated by increased puc-lacZ expression. When UAS-odd ${ }^{R N A i}$ was expressed in puc-lacZ background (to monitor puc expression levels), we observed increased levels of pupal lethality with da-, en- and pnr-GAL4 crosses. Particularly, no adult flies emerged when UAS-odd ${ }^{R N A i}$ was expressed with $d a-$ and en-GAL4 drivers. puc-lacZ is heterozygous for puc and increase in puc-lacZ is an indication of negative feedback regulation. Therefore, enhancement in the phenotype in pucheterozygous background (puc-lacZ) further confirms that the defect is due to enhanced JNK activity. There is a possibility that Odd suppresses puc expression, which then implies that the knock down of the former should activate the latter. If so, we expect that JNK signaling would be down-regulated rather than up-regulated as we have proposed here. In such a scenario, we expect thorax closure phenotype when Odd is knocked down using odd-GAL4 driver similar to the phenotype caused by the expression of dominant negative Bsk using the same GAL4 driver. Neither we observed such a phenotype nor any change in the cell morphology of medial edge cells suggesting that Odd does not act directly on puc. The thorax closure phenotype associated with the knock-down of Odd is more likely due to ectopic activation of JNK pathway, probably in DP cells underlying the medial edge cells of the PM.

Increased JNK signaling in only the presumptive notum cells as induced with down regulation of Odd with pnr-Gal4 probably leads to increased cell migration and the two halves of the thorax seem to move into each other and the notum appears narrower and in some cases the scutellum is barely seen. With en-Gal4, the number of flies showing defective thorax was not as high as seen with da-Gal4 or pnr-Gal4. This could possibly be because the domain of expression of en-Gal4 is the posterior compartment and not in the medial edge cells which play an important role in cell migration during thorax formation. As medial edge cells are the ones which fuse with the opposing wing discs along the anterior-dorsal edges, JNK signaling may provide the guidance cues for proper fusion. Since da-Gal4 is expressed in all cells of the wing disc including the medial edge cells, down regulation of Odd caused increased JNK activity in the entire wing disc which, probably drives the medial edge cells to fuse in an uncoordinated fashion that ultimately leads to defective phenotypes in adults and in severe cases also leads to pupal lethality. The results presented here thus, suggests the importance of restricting JNK signaling only to medial edge cells of the PM for proper thorax closure.

Our data provides evidence to add Odd to the growing list of proteins directly or indirectly involved in thorax closure. Further analysis is required both at the loss- and gain-of-function levels and also in identifying proteins that override the effect of Odd in the medial edge cells to keep JNK activity on. Bsk, which forms the core of the JNK signaling is regulated by the transcription factor dNF-Y. RNAi mediated down regulation of dNF-Y resulted in thorax closure defects owing to reduced level of Bsk (Yoshioka et al., 2008). odd is also one such transcriptional regulator that encodes a zinc finger protein, which has been reported to directly repress the segment polarity genes en and $w g$ in the early embryo. The C-terminus of odd-skipped has a functional eh-1 like sequence that recruits Groucho (grou) and mediates this repression (Goldstein, et al., 2005). However, further analysis is required to understand the precise mechanism by which Odd regulates JNK activity.

\section{Materials and Methods}

\section{Fly stocks}

Targeted misexpression of transgenes was achieved using the UASGAL4 system (Brand and Perrimon, 1993). The GAL-4 drivers used were odd-GAL4 and Ubx-GAL4 (Pallavi and Shashidhara, 2003), da-GAL4 (Wodarz et al., 1995), en-GAL4 (Brand and Perrimon, 1993), MS1096-GAL4 (Capdevila et al., 1994), puc-Gal4 (Pastor-Pareja et al., 2004) pnr-GAL4 and ap-GAL4 (Calleja et al., 1996). UAS lines used were UAS-nuclear lacZ (Brand and Perrimon, 1993), UAS-Ubx (Castelli-Gair et al., 1994), UAS-mcd8GFP (Lee and Luo, 1999), UAS-bsk (Mlodzik, Flybase reference 2005.10), UAS-bsk ${ }^{D N}$ (Adachi-Yamada, T. Flybase reference 2001.12.10) and UAS-PUC EP3194 (S Noselli, unpublished). LacZ enhancer trap lines used were ap-lacZ (Diaz-Benjumea and Cohen, 1993), puc-lacZ (Martin-Blanco et al., 1998) and odd-lacZ (Schroeder et al., 2004). All the experiments were performed at $25^{\circ} \mathrm{C}$ unless mentioned otherwise, with the wild type Canton-S flies serving as the control.

Lineage-tracing technique is essentially as described by Weigmann and Cohen, (1999). lacZclones were generated by crossing Actin5C >stop >lacZ to UAS-FLP;odd-GAL 4 at $25^{\circ} \mathrm{C}$. Third larval instar wing discs were stained for lac $Z$ with anti- $\beta$-galactosidase antibodies. Ubx clones were generated by FLP/FRT techniques (Xu and Rubin, 1993) using p[FRT] 82BUbx ${ }^{1}$ (Shashidhara et al.,1999).

\section{Generation of transgenic flies}

UAS-odd ${ }^{R N A i}$ transgenic flies were generated for odd. Primers were designed to amplify the sequences from 1441-1670 from the cDNA clone RE48009 encoding the transcript of odd. The amplified fragment was sub cloned into pUAST-symp Vector. The construct was sequence verified and injected into the embryos of $w 1118$ fly strain along with a transient source of transposase for integration. The loss of function of odd was confirmed by staining for odd antibody in the wing discs expressing UAS-odd ${ }^{\text {RAA }}$.

\section{Larval dissections and immunohistochemistry}

Immunohistochemical staining was performed essentially as described by Patel et al., (1989). The primary antibodies used were polyclonal anti$\beta$-galactosidase (in house, CCMB), anti-Armadillo (Riggleman et al., 1990) anti-Ubx (White and Wilcox, 1984), and anti Odd-skipped (Kosman et al., 1998; gift by S. Small) at concentrations of 1:250, 1:100, 1:100 and 1:20 respectively. Secondary antibodies conjugated to fluorophores (Alexa dyes) were obtained from Molecular Probes, USA and used at a concentration of 1:1000. Images were obtained on Zeiss Apotome microscope or Zeiss LSM/Meta Confocal or a Zeiss Multiphoton microscope.

\section{Acknowledgements}

We thank Bloomington Stock Centre, Development Studies Hybridoma Bank and S. Small for fly stocks and antibodies, SK Pallavi for wingdisc schematic, and A Aritakula-Mehla for expression patterns of GAL4 drivers and members of LSS and SN labs for discussions and help. We thank Indo-French Centre for the Promotion of Advanced Research (IFCPAR) for a joint research grant to LSS and SN and Department of Atomic Energy (Government of India) for a research grants to LSS.

\section{References}

AGNES, F., SUZANNE, M., NOSELLI, S. (1999). The Drosophila JNK pathway controls the morphogenesis of imaginal discs during metamorphosis. Development 126: 5453-5462.

BRAND, A. H. and PERRIMON, N. (1993). Targeted gene expression as a means of altering cell fates and generating dominant phenotypes. Development 118:401-415.

CALLEJA, M., MORENO, E., PELAZ, S. and MORATA, G. (1996). Visualization of gene expression in living adult Drosophila. Science 274: 252-255. 
CAPDEVILA, J., PARIENTE, F., SAMPEDRO, J., ALONSO, J.L. and GUERRERO, I., (1994). Subcellular localization of the segment polarity protein Patched suggests an interaction with the Wingless receptor complex in Drosophila embryos. Development 120: 987-998.

CASTELLI-GAIR, J., GREIG, S., MICKLEM, G. and AKAM, M. (1994). Dissecting the temporal requirements for homeotic gene function. Development 120: 1983-1995.

CHANG, L. and KARIN, M., (2001). Mammalian MAP kinase signalling cascades. Nature 410: 37-40.

CHO, K.-O., CHERN, J., IZADDOOST, S. and CHOI, K.-W. (2000). Novel signaling from the peripodial membrane is essential for eye disc patterning in Drosophila. Cell 103: 331-342.

DAVIS, R.J. (2000). Signal transduction by the JNK group of MAP kinases. Cell 103: $239-252$

DIAZ-BENJUMEA, F. J. and COHEN, S. M., (1993). Interaction between dorsal and ventral cells in the imaginal disc directs wing development in Drosophila. Cell 75:741 -752.

FRISTROM, D. and FRISTROM J.W. (1993). The metamorphic development of adult epidermis. In The Development of Drosophila Melanogaster. (ed. Bate, M. and Martinez Arias, M.). Cold spring Harbor Laboratory Press.

GIBSON, M. C. and SCHUBIGER, G. (2000). Peripodial cells regulate proliferation and patterning of Drosophila imaginal discs. Cell 103: 343-350.

GIBSON, M. C., LEHMAN, D. A. and SCHUBIGER, G., (2002). Lumenal transmission of Decapentaplegic in Drosophila imaginal discs. Dev Cell 3: 451-460.

GLISE, B., BOURBON, H. and NOSELLI, S., (1995). Hemipterous encodes a novel Drosophila Map kinase kinase required for epithelial cell sheet movement. Cell 83: 451-461.

GOLDSTEIN, R.E., COOK, O., DINUR, T., PISANTE, A., KARANDIKAR, U.C., BIDWAI, A. and PAROUSH, Z., (2005). An eh1-Like Motif in Odd-skipped Mediates Recruitment of Groucho and Repression In Vivo. Mol Cell Biol 25: 10711-10720

IP, Y. T. and DAVIS, R.J. (1998). Signal transduction by the C-Jun N-terminal kinase (JNK)-from inflammation to development. Curr Opin Cell Biol 10: 205-219.

ISHIMARU, S., UEDA, R., HINOHARA, Y., OHTANI, M. and HANAFUSA1, H., (2004). PVR plays a critical role via JNK activation in thorax closure during Drosophila metamorphosis. EMBO J 23: 3984-3994.

KOSMAN, D., SMALL, S., REINITZ, J., (1998). Rapid preparation of a panel of polyclonal antibodies to Drosophila segmentation proteins. Dev Genes Evol 208: 290-294.

LEE, T. and LUO, L., (1999). Mosaic analysis with a repressible cell marker for studies of gene function in neuronal morphogenesis. Neuron 22: 451-461.

MARTIN-BLANCO, E., GAMPELA., RING J., VIRDEE, K., KIROV, N., TOLKOVSKY, A.M. and MARTINEZ-ARIAS, A., (1998). Puckered encodes a phosphatase that mediates a feed back lop regulating JNK activity during dorsal closure in Drosophila. Genes Dev 12: 557-570.

MARTIN-BLANCO, E., PASTOR-PAREJA, J.C. and GARCIA-BELLIDO, A., (2000). JNK and decapentaplegic signaling control adhesiveness and cytoskeleton dynamics during thorax closure in Drosophila. Proc Natl Acad Sci USA. 97: 7888-7893.

MILNER., M.J., BLEASBY, A.J. and KELLY, S.L. (1984). The role of the peripodial membrane of leg and wing imaginal discs of Drosophila Melanogaster during evagination and differentiation in vitro. Whilelm Roux's Archiv Dev Biol 193: 180-186.

NOSELLI, S. and AGNES, F. (1999). Roles of the JNK signaling pathway in Drosophila Morphogenesis. Curr Opin Genet Dev 9: 466-472.
PALLAVI, S.K. and SHASHIDHARA, L.S. (2003). Egfr/Ras pathway mediates interactions between peripodial and disc proper cells in Drosophila wing discs. Development 130: 4931-4941.

PALLAVI, S.K. and SHASHIDHARA, L.S. (2005). Signaling interactions between squamous and columnar epithelia of Drosophila wing disc. J Cell Science 118 3363-3370.

PASTOR-PAREJA, J.C., GRAWE, F., MARTIN-BLANCO, E. and GARCIA-BELLIDO A. (2004). Invasive cell behavior during Drosophila imaginal disc eversion is mediated by the JNK signaling cascade. Dev Cell 7: 387-399.

PATEL, N.H., MARTIN-BLANCO, E., COLEMAN, K.G., POOLE, S.J., ELLIS, M.C. KORNBERG, T.B. and GOODMAN, C.S. (1989). Expression of Engrailed proteins in arthropods, annelids, and chordates. Cell 58: 955-968.

RIESGO-ESCOVAR, J.R., JENNI, M., FRITZ, A. and HAFEN, E. (1996). The Drosophila jun-N-terminal kinase is required for cell morphogenesis but not for DJun-dependent cell fate specification in the eye. Genes Dev 10: 2759-2768.

RIGGLEMAN, B., SCHEDL, P. and WIESCHAUS, E. (1990). Spatial expression of the Drosophila segment polarity gene armadillo is posttranscriptionally regulated by Wingless. Cell 63: 549-560.

SCHROEDER, M.D., PEARCE, M., FAK, J., FAN, H., UNNERSTALL, U., EMBERLY, E., RAJEWSKY, N., SIGGIA, E.D. and GAUL, U. (2004). Transcriptional control in the segmentation gene network of Drosophila. PLOS BIOL. 2(9): e271.

SHASHIDHARA, L. S., AGRAWAL, N., BAJPAI, R., BHARATHI, V. and SINHA, P. (1999). Negative Regulation of Dorso-Ventral Signaling by the Homeotic Gene Ultrabithorax during Haltere Development in Drosophila. Dev Biol 212: 491-502.

SLUSS, H.K., HAN, Z., BARRETT, T., DAVIS, R.J. and IP, Y.T. (1996). A JNK signal transduction pathway that mediates morphogenesis and an immune response in Drosophila. Genes Dev 10: 2745-2758.

SUZANNE, M., PERRIMON, N. and NOSELLIS., (2001). The Drosophila JNK pathway controls the morphogenesis of the egg dorsal appendages and micropyle. Dev Biol 237: 282-294.

TATENO, M., NISHIDA, Y. and ADACHI-YAMADA, T. (2000). Regulation of JNK by Src during Drosophila development. Science 287: 324-327.

WEIGMANN, K. and COHEN, S. M. (1999). Lineage tracing cell born in different domains along the P/D axis of the developing Drosophila leg. Development 126: 3823-3830.

WESTON, C.R. and DAVIS, R.J. (2002). The JNK signal transduction pathway. Curr Opin Genet Dev 12: 14-21.

WHITE, R. A. H. and WILCOX, M. E. (1984). Protein products of the Bithorax complex in Drosophila. Cell 39: 163-167.

WODARZ, A., HINZ, U., ENGELBERT, M. and KNUST, E., (1995). Expression of crumbs confers apical character on plasma membrane domains of ectodermal epithelia of Drosophila. Cell 82: 67-76.

XU, T. and RUBIN, G.M. (1993). Analysis of genetic mosaics in developing and adult Drosophila tissues. Development 117: 1223-1237.

YOSHIOKA, Y., SUYARI, O. and YAMAGUCHI., M. (2008). Transcription factor NF-Y is involved in regulation of the JNK pathway during Drosophila thorax development. Genes Cells 13: 117-130.

ZEITLINGER J. and BOHMANN, D. (1999). Thorax closure in Drosophila: Involvement of Fos and the JNK pathway. Development 126: 3947-3956. 


\section{Further Related Reading, published previously in the Int. J. Dev. Biol.}

Both jnk and apoptosis pathways regulate growth and terminalia rotation during Drosophila genital disc development Sergio Benitez, Claudia Sosa, Nicolás Tomasini and Ana Macías Int. J. Dev. Biol. (2010) 54: 643-653

Origin and proliferation of blastema cells during regeneration of Drosophila wing imaginal discs Manel Bosch, Jaume Baguñà and Florenci Serras Int. J. Dev. Biol. (2008) 52: 1043-1050

Role of Jun N-terminal Kinase (JNK) signaling in the wound healing and regeneration of a Drosophila melanogaster wing imaginal disc

Jaakko Mattila, Leonid Omelyanchuk, Satu Kyttälä, Heikki Turunen and Seppo Nokkala Int. J. Dev. Biol. (2005) 49: 391-399

The ash2 gene is involved in Drosophila wing development Montserrat Amorós, Montserrat Corominas, Peter Deák and Florenci Serras Int. J. Dev. Biol. (2002) 46: 321-324

A screen for genes expressed in Drosophila imaginal discs Offer Gerlitz, Denise Nellen, Michael Ottiger and Konrad Basler Int. J. Dev. Biol. (2002) 46: 173-176

Regulatory control of signal transduction during morphogenesis in Drosophila E Martín-Blanco

Int. J. Dev. Biol. (1998) 42: 363-368

ISI Impact Factor $(2010)=2.86$
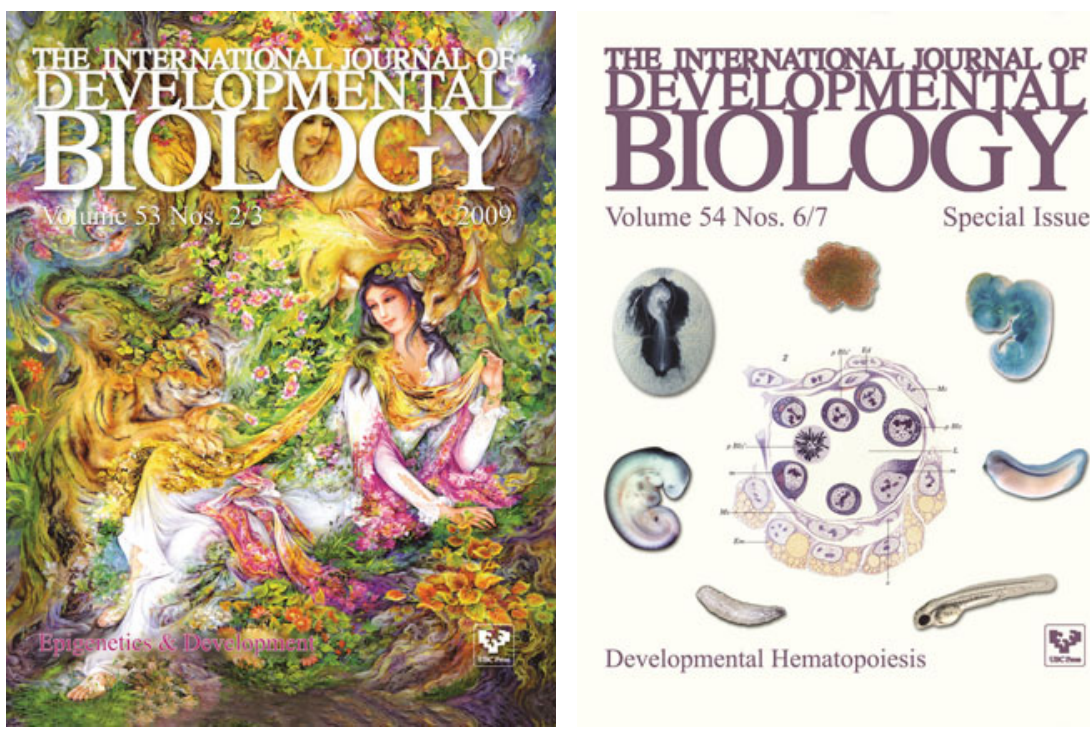

Volume 54 Nos. $6 / 7$

Special Issue
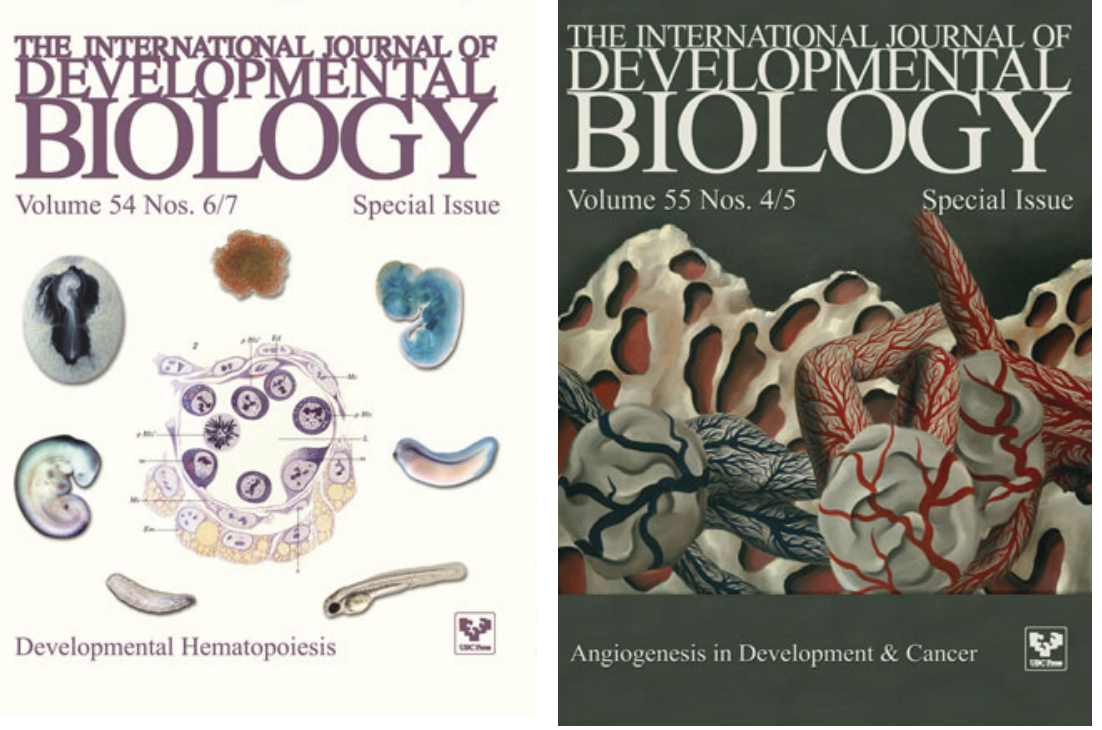